\title{
Small-Action Resonance in Hamiltonian Systems and Redistribution of Energetic Ions in Tokamaks
}

\author{
Yu. V. Yakovenko, Ya. I. Kolesnichenko, V. V. Lutsenko, \\ Scientific Centre "Institute for Nuclear Research", Kyiv, 252680, Ukraine \\ and
}

R. B. White

Princeton Plasma Physics Laboratory, P.O.Box 451, Princeton, NJ, 08543, USA

(May 28, 1999)

\begin{abstract}
It has been found that an arbitrary small perturbation in an integrable Hamiltonian system typically leads to driven resonance in the regions of the phase space where at least one of the action variables is sufficiently small. In particular, such a small-action resonance is shown to play a dominant role in the sawtooth-crash-induced disappearance of a strongly localized $\gamma$-ray and neutron emitting region in a tokamak plasma, which was observed experimentally.
\end{abstract}

05.45.-a, 52.55.Fa, 52.65.Cc, 52.25.Fi

Typeset using REVTEX 
Resonant interaction of particles with weak symmetry breaking perturbations produced by, e.g., the presence of electromagnetic or other waves is known to play an important role in physics. It involves those particles which satisfy, at least approximately, the resonance condition. The latter can be written as

$$
\sum_{i} n_{i} \omega_{i}=\Omega
$$

where $\omega_{i}$ are the frequencies of the particle motion along the canonical angles $\phi_{i}, i=1 \ldots N, \Omega$ is the perturbation frequency, and $n_{i}$ are integers. In case Eq. (1) is satisfied for the unperturbed particle motion, it determines certain resonance surfaces in the phase space (resonant tori). In the vicinity of the resonant tori a small perturbation leads to destruction of the invariant tori of the unperturbed motion and the appearance of resonance islands, which we will refer to as "conventional islands" (CI), see, e.g., Ref. [1]. When the perturbation amplitude exceeds a certain threshold, the dependence of the frequencies $\omega_{i}$ on the perturbation amplitude may result in driven (forced) resonances. This was found by Karney [2] (see also [3]), who considered plasma heating by lower hybrid waves, and White [4], who studied the magnetic-ripple-induced diffusion of energetic ions in tokamaks. Driven resonance islands [2-4] appear in regions of the phase space where they were absent for perturbations below the threshold.

In this Letter we will show that driven resonances and concomitant domains where particles are trapped by the perturbation may exist even without an amplitude threshold. We will refer to them as "small-action resonances" (SAR) and "small-action resonance domains" (SARD) because they appear in the parts of phase space where one or more of the action variables is close to zero. To demonstrate this, we will consider the behavior of energetic ions with the orbits trapped poloidally in tokamaks with sawtooth oscillations. This study is of importance in connection with the sawtooth-crash-induced disappearance of the so-called "hot spot" observed on the Joint European Torus (JET) [5].

We begin our consideration with a linear oscillator in the presence of a small external force, which closely models the trapped ions. Such an oscillator can be described by the Hamiltonian

$$
H(p, q, t)=\frac{p^{2}}{2 M}+\frac{M \omega^{2} q^{2}}{2}+f q \cos (\Omega t)
$$


where $q, p$, and $M$ are the particle coordinate, momentum, and mass; $\omega$ is the frequency of free oscillations; $f$ and $\Omega$ are the amplitude and frequency of the perturbing force. Proceeding to the action-angle variables of the unperturbed system, $J$ and $\phi$, with the canonical transformation given by $q=(2 J / M \omega)^{1 / 2} \cos \phi, p=(2 M \omega J)^{1 / 2} \sin \phi$, we write Eq. (2) as

$$
\begin{aligned}
H(J, \phi, t)= & \omega J+\alpha J^{1 / 2}[\cos (\phi-\Omega t) \\
& +\cos (\phi+\Omega t)]
\end{aligned}
$$

where $\alpha=f(2 M \omega)^{-1 / 2}$. It is convenient to introduce the variables

$$
\hat{J}=J, \hat{\phi}=\phi-\Omega t
$$

instead of $J, \phi$ because then the equation of the conservation of the perturbation phase has the simple form $\dot{\hat{\phi}}=0$. The transformation is made with the generating function $S_{1}(\hat{J}, \phi, t)=$ $(\phi-\Omega t) \hat{J}$, giving the new Hamiltonian (a non-resonance term is omitted);

$$
\hat{H}(\hat{J}, \hat{\phi}, t)=\hat{\omega} \hat{J}+\alpha \hat{J}^{1 / 2} \cos \hat{\phi}
$$

where $\hat{\omega}=\omega-\Omega$. Letting $x=\hat{J}^{1 / 2} \cos \hat{\phi}$ and $y=\hat{J}^{1 / 2} \sin \hat{\phi}$, the level contours of $\hat{H}$ are easily seen to be concentric circles shifted to the center $x=-\alpha / 2 \omega$. This produces a domain where the particle trajectories are trapped with respect to the perturbation phase $(\pi / 2 \leq \hat{\phi} \leq 3 \pi / 2)$, i.e., a SARD. The presence of particles trapped in the perturbation is a common feature of SARD, CI and islands found in Refs. [2,4]. The appearance of SARD, in contrast to CI, does not result from a change of the topology of the phase portrait of the system; the invariant tori of the particle motion are shifted rather than destroyed, and SARD has one fixed point [located at $\left.\hat{J}=\alpha^{2} /\left(4 \hat{\omega}^{2}\right), \hat{\phi}=\pi\right]$, whereas the islands have additional fixed points.

The fixed point in Eq. (5) satisfies Eq. (1), which can be written as $\dot{\hat{\phi}}=0$ or as

$$
\hat{\omega}+\frac{\alpha}{2} \hat{J}^{-1 / 2} \cos \hat{\phi}=0
$$

It follows from Eq. (6) that the term associated with the external force is essential in the neighborhood of $\hat{J}=0$ even when the perturbation amplitude is arbitrarily small. This means that SARD exists for arbitrarily small amplitude of the perturbation. Note that the absence 
of the threshold for the existence of SARD also follows from the fact that SARD is produced by simple shift of the vicinity of the O-point of the unperturbed system.

Let us generalize the Hamiltonian (5) as

$$
\hat{H}=H_{0}(\hat{J})+U(\hat{J}) \cos \hat{\phi}
$$

where the first term is the Hamiltonian of the unperturbed motion, and the second term represents the perturbation, $U$ being an arbitrary function of $\hat{J}$. Equation (1) then takes the form

$$
\hat{\omega}+\frac{d U}{d \hat{J}} \cos \hat{\phi}=0,
$$

where $\hat{\omega}=d H_{0} / d \hat{J}$. We see that the perturbation modifies the frequency of motion and may make resonance possible even when it is absent in the unperturbed system, i.e. $\hat{\omega} \neq 0$ may lead to driven resonances. This takes place in two cases. First, driven resonances occur when the perturbation amplitude exceeds a certain threshold magnitude: $U(\hat{J})>\hat{J} \hat{\omega}(d \ln U / d \ln \hat{J})^{-1}$. For a small perturbation, $U / H_{0} \sim U /(\hat{\omega} \hat{J}) \ll 1$, this condition can be satisfied only when the perturbation is characterized by large gradients, $d \ln U / d \ln \hat{J} \gg 1$. Then resonance islands similar to those of Refs. [2-4] are formed, which have topology different from that of CI and SARD as well. Second, Eq. (8) is satisfied when $d U / d \hat{J}$ has a singularity [like the Hamiltonian given by Eq. (5)], in which case there is no amplitude threshold. This is possible only at $J=0$ (when SAR occurs) if the Hamiltonian is an analytical function of the coordinates and the momenta. Note that the existence of SAR is a common feature of Hamiltonian systems when one of the action variables is sufficiently small. Indeed, $J=\oint p d q$ is proportional to the square of the contour size when the contour shrinks to a point, which typically leads to the singularity $J^{-1 / 2}$ in $\partial H / \partial J$ at $J \rightarrow 0$.

In spite of the described differences between SARD and CI, they are related in the sense that they may turn into each other when parameters of the system change. To show this, we introduce the non-linear term $\gamma \hat{J}^{2}$ into Eq. (5). Proceeding to variables $\tilde{J}=(\gamma / \alpha)^{2 / 3} \hat{J}$, $\tilde{J}_{r}=-\hat{\omega} \alpha^{-2 / 3} \gamma^{-1 / 3} / 2$, we write the normalized Hamiltonian as

$$
\tilde{H}=\left(\tilde{J}-\tilde{J}_{r}\right)^{2}+\tilde{J}^{1 / 2} \cos \hat{\phi},
$$


where $\tilde{J}_{r}$ is the resonance action in the absence of the perturbation. One can see that, when $\tilde{J}_{r}<0$ (i.e., when there is no resonance in the unperturbed system), Eq. (9) yields a a plot which coincides with that of Eq. (5) in the vicinity of $\tilde{J}=0$ but differs from it at large $\tilde{J}$ by a decrease of the orbit displacement with $\tilde{J}$. This means that our conclusions obtained above by neglecting non-linearity in the unperturbed motion are qualitatively valid also for non-linear systems but only near the resonance. On the other hand, when $J_{r} \gg 1$, Eq. (9) is reduced to the standard Hamiltonian [1] and, thus, describes CI. This becomes clear if we note that the island width is $\Delta \tilde{J} \sim \tilde{J}_{r}^{1 / 4}$. Hence, the variation of $\tilde{J}$ in the last term of Eq. (9) can be neglected at distances greater than the island width. In the intermediate case, $\tilde{J}_{r} \sim 1$, there are two critical magnitudes: $\tilde{J}_{r 1}=3 / 4$ and $\tilde{J}_{r 2}=2^{1 / 3} \tilde{J}_{r 1}$, cf. [6]. When $\tilde{J}<\tilde{J}_{r 1}$, the surfaces are still topologically concentric circles (but SARD becomes bean-shaped, and the resonance cannot be called driven). At $\tilde{J}_{r}>\tilde{J}_{r 1}$, a separatrix with an X-point appears (see Fig. 1), SARD occupying a part of the arising island. When $\tilde{J}_{r}=\tilde{J}_{r 2}$, SARD occupies the whole interior of the separatrix and is transformed into an island, which differs from CI only by being asymmetric with respect to $\tilde{J}_{r}$. Note that CI may turn into SARD when the perturbation amplitude increases. This follows from the fact that $\tilde{J}_{r} \propto \alpha^{-2 / 3}$.

Now we proceed to analysis of a system with two degrees of freedom,

$$
\begin{gathered}
H\left(J_{\theta}, J_{\phi}, \theta, \phi\right)=\omega_{\theta} J_{\theta}+\omega_{\phi} J_{\phi} \\
+\alpha J_{\theta}^{1 / 2} \cos (\theta-n \phi+\Omega t),
\end{gathered}
$$

where $\theta$ and $\phi$ are angular coordinates; $J_{\theta}$ and $J_{\phi}$ are the corresponding action variables, $J_{\theta}$ being close to zero; $\omega_{\theta}$ and $\omega_{\phi}$ are the frequencies of the unperturbed motion; $\alpha, \Omega$, and $n$ are the perturbation amplitude, frequency, and mode number, respectively. We perform a canonical transformation to the new actions and angles, $\hat{J}_{\theta}, \hat{J}_{\phi}, \hat{\theta}$, and $\hat{\phi}$, so that $\dot{\hat{\phi}}=0$ corresponds to resonant motion, which can be done with the generating function $S_{2}\left(\hat{J}_{\theta}, \hat{J}_{\phi}, \theta, \phi\right)=\hat{J}_{\theta} \theta / n+$ $\hat{J}_{\phi}(\theta-n \phi+\Omega t)$. We obtain the following new Hamiltonian

$$
\begin{array}{r}
\hat{H}\left(\hat{J}_{\theta}, \hat{J}_{\phi}, \hat{\theta}, \hat{\phi}\right)=\hat{\omega}_{\theta} \hat{J}_{\theta}+\hat{\omega}_{\phi} \hat{J}_{\phi} \\
+\alpha\left(\frac{\hat{J}_{\theta}}{n}+\hat{J}_{\phi}\right)^{1 / 2} \cos \hat{\phi}
\end{array}
$$


where $\hat{\theta}=\theta / n, \hat{\phi}=\theta-n \phi+\Omega t, \hat{J}_{\theta}=n J_{\theta}+J_{\phi}, \hat{J}_{\phi}=-J_{\phi} / n, \hat{\omega}_{\theta}=\omega_{\theta} / n, \hat{\omega}_{\phi}=\omega_{\theta}-n \omega_{\phi}+\Omega$. As $\partial \hat{H} / \partial \hat{\theta}=0, \hat{J}_{\theta}$ is a constant of motion. The level contours of the Hamiltonian (11) for a given $\hat{J}_{\theta}$ are shifted circles in the coordinates $\left(\hat{J}_{\phi}-\hat{J}_{\phi 0}\right)^{1 / 2} \cos \hat{\phi},\left(\hat{J}_{\phi}-\hat{J}_{\phi 0}\right)^{1 / 2} \sin \hat{\phi}$, where $\hat{J}_{\phi 0}=-\hat{J}_{\theta} / n$ is the value of $\hat{J}_{\phi}$ that corresponds to $J_{\theta}=0$. Thus, they are similar to those of the Hamiltonian (5), and SARD arises near $J_{\theta}=0$.

An example of the described two-dimensional picture is the motion of energetic ions in a tokamak in the presence of sawtooth oscillations. For them one can use the poloidal and toroidal canonical angles, $\theta$ and $\phi$ introduced in Refs. [7-9]; the corresponding actions are the toroidal magnetic flux through the particle orbit, $J_{\theta}$, and the canonical angular momentum $J_{\phi}$. We proceed from the fact that, when the ion energy, $\mathcal{E}$, exceeds the critical magnitude, $\mathcal{E}_{\text {crit }}$ [10], the resonances

$$
s \omega_{\theta}=n \omega_{\phi},
$$

where $\omega_{\theta}$ and $\omega_{\phi}$, the bounce and precession frequencies, respectively, and the integer $s$, play an important role in the transport of trapped particles during sawtooth crashes, leading to superbanana orbits $[11,9]$. As $\omega_{\theta}$ exceeds $\omega_{\phi}$, CI and concomitant superbananas arise only for $n>1$ (when $s \neq 0$ ). Because of this, the $n=1$ harmonic, which is dominant in sawtooth crashes, cannot affect the particles by creating CI. However, one can expect that certain groups of energetic ions will be affected by this mode through SAR. These are the particles with the Otype stagnation orbits or orbits close to them, i.e., trapped poloidally but untrapped toroidally, for which $J_{\theta}$ is small. According to Ref. [5], such particles $\left({ }^{3}\right.$ He minority ions accelerated during ion cyclotron resonance frequency heating up to energies of several $\mathrm{MeV}$ ) were responsible for the "hot spot" observed in JET experiments - a strongly localized gamma-ray and neutron emitting region in the plasma core, which disappeared after crashes.

In previous works of the authors $[9,12]$, when SAR was not known, the disappearance of the hot spot was attributed to the motion of the hot-spot ions along CI produced by $n=2,3$ modes. These modes appear in the nonlinear stage of the $m=n=1$ instability resulting in the crash ( $m$ is the poloidal mode number). However, the modes with $n>1$ are significant for a time less than the crash duration, and their amplitudes are relatively small. 
In order to investigate the effect of the $m=n=1$ mode on the hot-spot ions, Poincaré maps of their motion were generated. The drift equations [8] and the code of Ref. [9] were used to find intersections of particle trajectories with positive parallel velocity with the equatorial plane of the torus. The results in the variables $\left(\hat{J}_{\phi}-\hat{J}_{\phi 0}\right)^{1 / 2} \cos \varphi,\left(\hat{J}_{\phi}-\hat{J}_{\phi 0}\right)^{1 / 2} \sin \varphi$, where $\hat{J}_{\phi}=-J_{\phi}, \hat{J}_{\phi 0}$ is the value of $\hat{J}_{\phi}$ corresponding to $J_{\theta}=0, \varphi$ is the toroidal coordinate equal to $-\hat{\phi}$ for the considered Poincaré map, are shown in Fig. 2. We observe that the particle trajectories form a SARD, as could be expected from Eq. (11). These orbits can be considered superbananas, as in the case of CI studied in Refs. [11,9]. The SAR-induced motion may result in considerable radial displacement of the ion from the unperturbed orbit, as is shown in Fig. 3 (the small degree of stochasticity is an irrelevant complication). Therefore, when the characteristic time of the change of the perturbation is of the order or less than the period of the superbanana motion (which is the case in the experiment), hot-spot ions with various initial $\varphi$ become dispersed after the crash almost over the whole sawtooth mixing region, which implies disappearance of the hot spot. The width of SARD exceeds that of CI produced by $n=2,3$ harmonics of the crash. Thus, it is SAR that is responsible for the expulsion of ions from the hot-spot region shown in Fig. 4.

In conclusion, we have shown that a perturbation with arbitrarily small amplitude typically produces a specific resonance domain (SARD) in the part of the phase space where one of the action variables is close to zero. The motion of energetic ions along SARD may explain the disappearance of a strongly localized emitting region ("hot spot") during sawtooth crashes in JET experiments [5].

The research described in this publication was made possible in part by the Award No. UP2290 of the Government of Ukraine and CRDF. The authors thank V.S. Marchenko for discussions. 


\section{REFERENCES}

[1] B.V. Chirikov, Phys. Reports 52, 265 (1979).

[2] C.F.F. Karney, Phys. Fluids 21, 1584 (1978).

[3] A.J. Lichtenberg, M.A. Lieberman, Regular and stochastic motion (Springer-Verlag, Berlin - Heidelberg - New York, 1983).

[4] R.B. White, Phys. Rev. E 58, 1774 (1998).

[5] O.N. Jarvis et al., Nucl. Fusion 36, 1513 (1996).

[6] I.A. Kotel'nikov and G.V. Stupakov, Sov. Phys. JETP 57, 555 (1983).

[7] A.N. Kaufman, Phys. Fluids 15, 1063 (1972).

[8] R.B. White and M.S. Chance, Phys. Fluids 27, 2455 (1984).

[9] Ya.I. Kolesnichenko, V.V. Lutsenko, R.B. White, and Yu.V. Yakovenko, Phys. Plasmas 5, 2963 (1998).

[10] Ya.I. Kolesnichenko and Yu.V. Yakovenko, Nucl. Fusion 36, 159 (1996).

[11] Ya.I. Kolesnichenko, V.V. Lutsenko, and Yu.V. Yakovenko, Phys. Plasmas 5, 729 (1998).

[12] Ya.I. Kolesnichenko, V.V. Lutsenko, R.B. White, Yu.V. Yakovenko, and S.J. Zweben, Extended Synopses. 17th IAEA Fusion Energy Conference (Yokohama, 1998), 360 (IAEA, Vienna, 1998). 


\section{FIGURES}

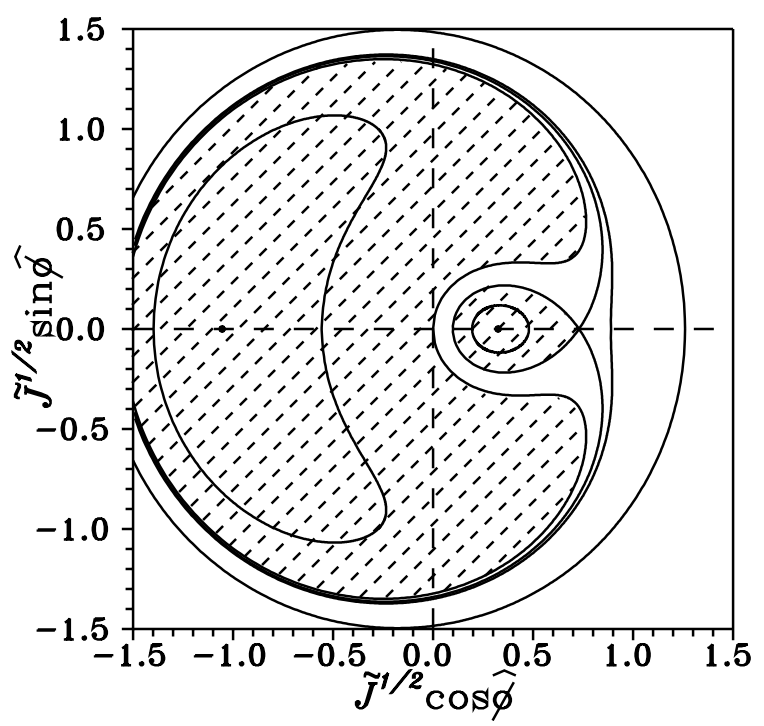

FIG. 1. Level contours of $\tilde{H}$ given by Eq. (9) with $\tilde{J}_{r}=7 / 8$. The resonance domains are hatched.

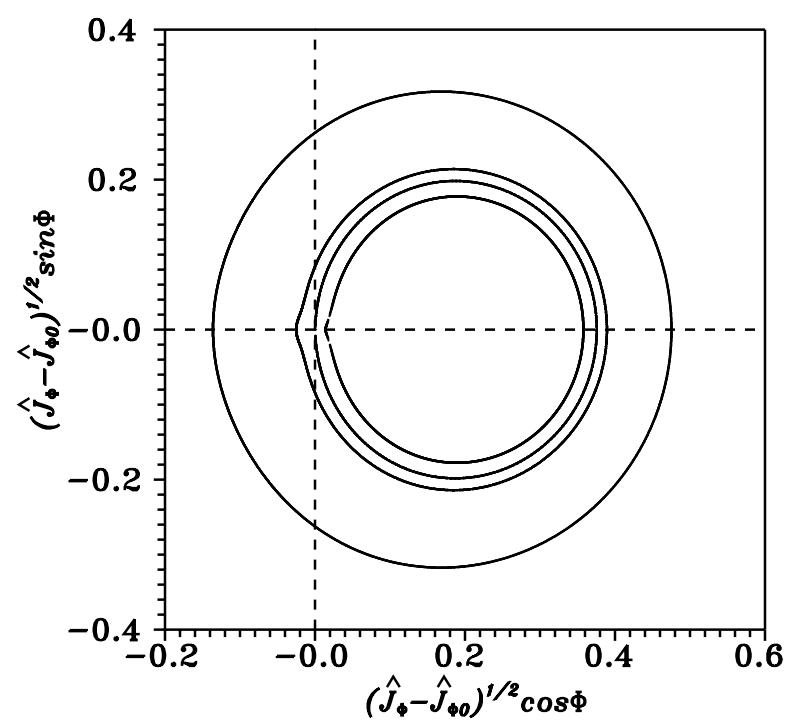

FIG. 2. The Poincaré map of the motion of $5 \mathrm{-MeV}$ hot-spot ions in JET under the $m=n=1$ perturbation of the magnetic field with the magnetic potential $[9] \Psi(r)=0.03 B_{0} r_{\text {mix }}^{2}\left(q_{0}^{-1}-1\right) r\left(1-r^{2}\right)$. Parameters: $R_{0}=3 \mathrm{~m}, B_{0}=3.5 \mathrm{~T}, q_{0}=0.8, r_{m i x}=60 \mathrm{~cm}, \lambda=1.0084$, where $B_{0}$ is the magnetic field at $r=0, R_{0}$ is the plasma major radius, $q_{0}$ is the safety factor at $r=0, r_{m i x}$ is the sawtooth mixing radius, $\lambda=\mu B_{0} / \mathcal{E}, \mu$ is the magnetic moment. 


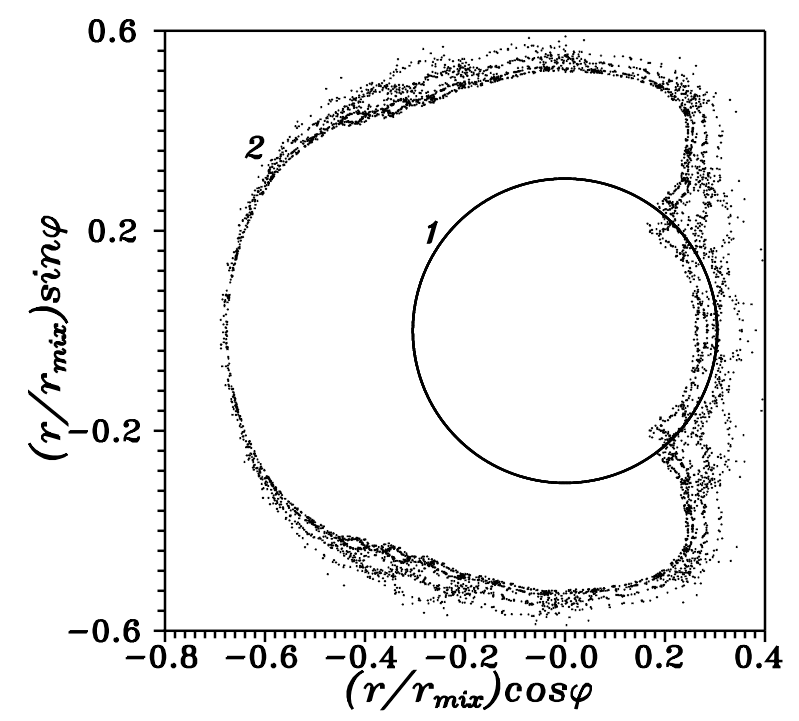

FIG. 3. The Poincaré maps of the motion of a hot-spot ion in JET: 1, in the absence of perturbations; 2 , in the presence of the $m=n=1$ perturbation (motion along SARD). The parameters are the same as in Fig. 2 except for a perturbation amplitude relevant to a sawtooth crash, $\Psi(r)=0.1 B_{0} r_{m i x}^{2}\left(q_{0}^{-1}-1\right) r\left(1-r^{2}\right)$.

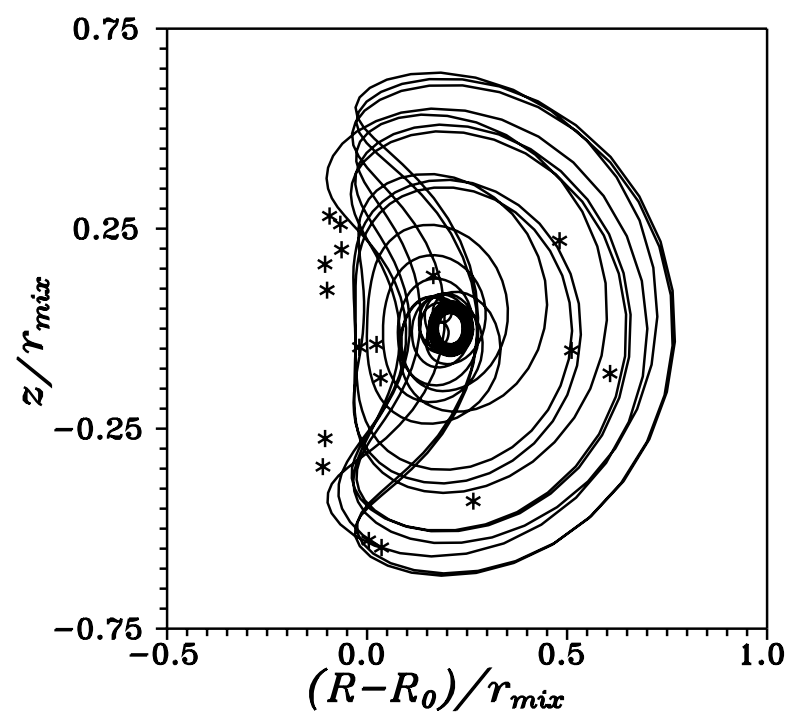

FIG. 4. Numerical modeling of the effect of a crash lasting for $10^{-4} \mathrm{~s}$ on hot-spot ions with $\mathcal{E}=5 \mathrm{MeV}$, based on the code OFSEF [12]. Notations: bold line, the pre-crash orbit of the ions; thin line, the orbit of one ion during the crash; asterisks, the post-crash locations of particles with various pre-crash angular coordinates; $R$ is the distance to the major axis of the torus; $z$ is the vertical coordinate. 